\title{
PRESENT STRATEGY IN THE DIAGNOSIS OF MENIERE'S DISEASE
}

\author{
M. PORTMANN, R. DAUMAN and D. PORTMANN
}

In spite of enormous amount of work carried out over the last 60 years, the origins of Meniere's disease still remain to a large extent mysterious.

It can, however, be defined from two points of view-clinical and physiopathological :

Clinical : The history of a case of Meniere's disease reveals a succession of acute attacks, of variable intensity, duration and frequency, comprising three major symptoms :

- Tinnitus, accompanied by a sensation of fullness in the ear,

- Sensorineural deafness,

- True vertigo accompanied by neurovegetative symptoms such as nausea and vomiting

It corresponds to a disorder of the inner ear ( $P$. Meniere) ${ }^{1)}$.

Physiopathological : The condition involves sudden attacks of high pressure in the endolymph, a concept first envisaged in 1926 by G. Portmann after a series of animal experiments ${ }^{2)}$ which then led to the operation involving the opening of the endolymphatic $\mathrm{sac}^{3)}$.

Twelve years later, Hallpike and Cairns ${ }^{4}$ provided histopathological proof of this high endolymph pressure.

At present, therefore, the only undisputed pathogenic factor is the existence of high endolymph pressure, but this has no doubt many varied causes -obstruction of the endolymphatic canal and sac for various reasons, autoimmune disorders, allergy crises, microcirculatory disorders of the stria vascularis, etc.

All cases of Meniere's disease involve high endolymph pressure, or hydrops, but not all cases of hydrops involve Meniere's disease. Hydrops is, in fact,

Clinique Universiaire d'O. R. L., Hopital

Pellegrin, Place A. Raba-Léon, 33076 Bordeaux, France found in many disorders, such as acoustic neurinoma (4\% of such cases in our statistics), labyrinthine syphilis, otosclerosis, etc.

Whatever the case, our diagnostic strategy comprises five different stages-clinical, audiovestibular, radiological, electrophysiological, and biological.

\section{CLINICAL EXAMINATION :}

This involves taking recognizance of the case history, which may be typical or atypical.

In typical cases, the attack comprises all three symptoms, occurring generally in young patients (with the highest frequency between 25 and 45 years). Attacks become increasingly frequent and decreasingly acute. Residual reception hypacusis between the attacks gradually worsens; the appearance of symptomps in the other ear is not exceptional and, in over $40 \%$ of these cases, leads to a bilateral pathology after several decades.

In a few, rare, atypical cases, these symptoms do not appear in the same order and/or degree of severity as in the classical description. In general, this due to the pathogenic involvment of other factors: vascular diseases, presbyacusis, etc.

\section{AUDIOVESTIBULAR EXAMINATION :}

Vestibular examination is very important. It involves electronystagmography and various other stimuli -opticokinetic, caloric, rotatory, etc.-and generally reveals a varying degree of hypoexcitability, though never total areflexia of the unilateral peripheral type.

The audiometric examination, however, is more important, especially in typical cases, with perception deafness involving the lower frequencies, recovery at $2000 \mathrm{~Hz}$, and the presence of marked recruitment. These symptoms fluctuate, and hearing may vary sometimes by between 20 and $40 \mathrm{~dB}$ in low frequencies at moments during the evolution of the condition. 


\section{RADIOLOGICAL EXAMINATION :}

Radiological examination is indispensable in all cases presenting an otological syndrome. In the particular case of a pathology involving the inner ear, there should be normal otoscopy and imaging with a view to checking the temporal bone, and above all the internal auditory canal and the cerebellopontine angle in order to eliminate any possibility of a tumour and in particular of an acoustic neurinona.

CT scanning, with a contrast injection, is therefore indispensable, and in the event of any suspicion or doubt, magnetic resonance imaging with a gadolinium injection will be preferable.

\section{ELECTROPHYSIOLOGICAL EXAMINATION :}

This is of fundamental importance and can be used to confirm the absence of any retrolabyrinthine lesion via B. E. R. A., or brainstem evoked response audiometry, and the presence of hydrops by detecting the summation potential, or S.P., using electrocochleography with an electrode on the promontorium, which is the most reliable method. It also provides an idea of the malleability and sensitivity of the phenomenon, and thus of the prognosis for possible treatment of the hydrops, via an electrocochleographic determination of the drop in the summation potential under the effect of glycerol, which has been studied particularly within our team by R. Dauman and his collbtorators, the conclusions of whose principal work I summarize here ${ }^{5)}$.

In typical cases of Meniere's disease, there is a high summation potential, both with bursts at low frequencies (1000 and $2000 \mathrm{~Hz}$, where the polarity of the summation potential is negative) and with bursts at high frequencies $(8000 \mathrm{~Hz}$, when the summation potential is positive). In cases of perception deafness of other sorts, however, such as ischemic labyrinthine disorder, there is little summation potential, whatever the frequency.

Between two groups of patients, respectively comprising 50 cases of Meniere's disease and 15 of perception deafness used for control purposes, there was a significant difference between the mean summation potentials at 1000,2000 and $8000 \mathrm{~Hz}$, despite a fairly wide scattering of the values (high standard deviation). Such scattering is an expression of the fact that there are cases of Meniere's disease with a high summation potential and others with loss. Correlation between the summation potential values and the clinical manifestations is not obvious. It can, however, be noted that cases of Meniere's disease with a high summation potential have not been considered as falling within the definition of the disease for as long as those where the summation potential is lower. Moreover, in those cases where the summation potential is markedly negative in th low frequencies, the action potential amplitude is also greater, which may be a sign of more favorable recording conditions.

The second ECochG criterion used to choose the type of operation is the evolution of the summation potential in the lower frequencies during the glycerol test. In a typical case of Meniere's disease, the summation potential drops gradually over the low frequencies, but does not change in the high frequencies. At the same time, the action potential does not decrease, and even tends to increase, in the low frequencies. Tlis drop in the summation potential in the high frequencies is found in $60 \%$ of cases of Meniere's disease. In our opinion, in shows both endolymphatic hydrops and the partial reversibility of the effects of such hydrops on the cochlea. No obvious relationship has appeared between the effects of glycerol on the summation potential and the following clinical characteristics : a feeling that the ear is blocked during the examination, still fluctuating hearing, the recent occurrence of an attack of vertigo, or the degree of deafness (both in the low and in the high frequencies). It therefore appears difficult to rely on the clinical manifestations when forecasting the effect of glycerol on the summation potential.

At a practical level, the data provided by the electrophysiological examination play a major role in the decision of whether or not to operate, and can now be added to the clinical criteria that have long been used in Bordeaux.

\section{BIOLOGICAL EXAMINATIONS :}

Once the diagnosis of Meniere's diseases is certain, an attempt must be made to determine any ascertainable etiological factors. Such a survey will involve neurological and allergic tests and examinations to see whether there may be viral, autoimmune or allergic causes, a cardiovascular examination, and a detailed blood metabolism analysis. 
In most cases, these tests prove negative, and provide no evidence to back up any particular course of therapeutic action.

From this data as a whole, two types of patient can be distinguished :

-Typical cases of Meniere's disease (a young patient untouched by any detectable systemic or metabolic disorder), and-Atypical cases, which often correspond to a disorder coming to the end of its course of development, an “old Meniere's disease”, or to truly abnormal forms. In these cases, there are non-characteristic attacks which cannot be considered true "Meniere's diseases", but which are referred to as "pseudo-Meniere's disease" or "Menieri form syndromes".

\section{IN CONCLUSION}

Our diagnostic strategy is based on five stages:

-Clinical : There is no Meniere's diseases without the characteristic syndrome.

-Audiovestibular: The vestibular, and above all the audiometric, aspects appear to be of fundamental importance.

- Radiological : This stage can eliminate the possibility of other local pathologies, particularly a retrolabyrinthine disorder.

-Electrophysiological : Detection of the S. P. is of fundamental importance for us. Electrocochleography can also be used to verify, moreover, the simultaneous taking of glycerol and the reversible character of phenomenon, and thus to draw up a prognosis for treatment.

-Finally biological, in order to try to detect any concomitant causal factors that can be treated, though unfortunately these are most commonly absent.

The patient should always receive medical treatment in the first instance. If this is unsuccessful, the endolymphatic sac may be opened. We usually consider two groups of criteria :

- Major criteria, of which at least two must be present if surgery is indicated : typical clinical symptoms, absence of any retrolabyrinthine pathology, pre- sence of a summation potential, improvement of the summation potential under the effect of glycerol, the latter crtiterion being essential among the two used.

-Minor criteria, including typical aspects of the vestibular examinations, a psychological and behavioural study, and the presence of concomitant biological causal factors.

In cases where these major criteria, particularly the absence of a reversal summation potentials with the glycerol test, are not present, it is preferable to perform an intracranial vestibular neurectomy directly.

In such cases, there is less likelihood that decompression of the sac will prove really useful.

\section{BIBLIOGRAPHY}

1) Ménière P : Pathologie auriculaire, maladies de l'oreille interne offrant les symptomes de la congestion cérébrale apoplectiforme. Gaz Med de Paris $16: 88-89,1861$

2 ) Portmann $G:$ Recherches sur la physiologie du sac et du canal endolymphatiques : valeur fonctionnelle de L'organe endolymphatique des selaciens. Compt Rend Soc du Biol 85 : 1070-1072, 1921

3) Portmann G: Vertigo, surgical treatment by opening the saccus endolymphaticus. Arch Otolaryngol $6: 309-319,1927$

4) Hallpike CS and Cairns H : Observations on pathology of Meniere's syndrome. J Laryngol 53 : 625-655, 1938

5 ) Dauman R, Darrouzet V, Bebear JP : Indications chirurgicales dans la maladie de Ménière-interet de l'electrocochleographie. Revue de Laryngologie $109: 249-250,1988$

Key words : Meniere's disease, electrocochleography, summating potential

Received and accepted: October 28, 1988

M. Portmann, M. D.

Clinique Universitaire d'O. R. L., Hopital

Pellegrin, Place A. Raba-Léon, 33076

Bordeaux, France 